CONFORMAL GEOMETRY AND DYNAMICS

An Electronic Journal of the American Mathematical Society

Volume 2, Pages 107-113

S 1088-4173(98)00024-1 (December 8, 1998)

\title{
CONFORMALLY CONVERTING CUSPS TO CONES
}

\author{
CHRISTOPHER M. JUDGE
}

\begin{abstract}
Conformal deformations of hyperbolic surfaces with conical singularities are shown to be real-analytic. The first nontrivial term in the power series expansion around a cusped surface is shown to be a multiple of the Eisenstein series $E_{2}$.
\end{abstract}

\section{INTRODUCTION}

Each finitely punctured Riemann surface has a unique complete hyperbolic metric of finite volume. With respect to this metric, each puncture has a neighborhood that can be isometrically embedded into the model hyperbolic cusp (see $\S 2$ ). It is natural to regard the model hyperbolic cusp as a hyperbolic cone with zero cone angle. In particular, the model cusp metric is an extremal member of a family of conformally equivalent hyperbolic cone metrics. With respect to natural coordinates on $S^{1} \times \mathbb{R}$, this family of model metrics is parametrized real-analytically by the cone angle (see $\S 2$ ). The main purpose of this paper is to prove that this holds for more general surfaces. Namely, we have Theorem 1: A hyperbolic surface with cusps belongs to a unique conformal deformation of hyperbolic surfaces with cones and this deformation is real-analytically parametrized by the cone angles.

Our proof of Theorem 1 is in the spirit on an implicit function argument contained in earlier work of M. Wolf [Wlf91] on degeneration of hyperbolic surfaces. In $\S 3$ we give a heuristic version of this argument and then make necessary modifications to the implicit function in preparation for the rigorous proof.

As further preparation, we define in $\S 4$ the Banach spaces that will act as domain and range for the implicit function. For these Hölder type spaces (on general Riemannian manifolds) we develop the usual potential theory including a compactness theorem and a Schauder estimate (Lemma 1). Then in $\S 5$, we use these spaces to prove Theorem 1.

In $\S 6$, we substitute the Maclaurin series provided by Theorem 1 into the prescribed curvature equation and obtain Theorem 2: The first nontrivial term is a multiple of the Eisenstein series $E_{2}$.

These results are part of the author's thesis [Jdg93]. The author thanks Scott Wolpert for suggesting the problem. We also acknowledge an obvious debt to the work found in [Wlf91]. An application of these results can be found in [Jdg95].

Received by the editors January 20, 1998 and, in revised form, November 16, 1998.

1991 Mathematics Subject Classification. Primary 30F10; Secondary 35J60, 53A30.

Key words and phrases. Eisenstein series, hyperbolic surface.

Manuscript preparation supported in part by NSF DMS 9304580 (IAS) and an NSF postdoctoral fellowship.

(C)1998 American Mathematical Society 


\section{From the MOdel CUSP TO THE MOdel CONE}

We give a general notion of a 2-dimensional metric cone. Let $d s$ be a (suitably smooth) element of arclength on the cylinder $\left.S^{1} \times\right] a, b[, a<b \in \hat{\mathbb{R}}$. We first suppose that the length, $\ell(t)$, of the circle $S^{1} \times\{t\}$ tends to zero as $t$ tends to $b$. Let $d(s, t)$ denote the (signed) distance between the circle $S^{1} \times\{s\}$ and $S^{1} \times\{t\}$. The rate of change in $\ell$ with respect to $d$ provides a notion of cone angle:

$$
\alpha=\lim _{t \rightarrow b}\left(\lim _{s \rightarrow t} \frac{\ell(s)-\ell(t)}{d(s, t)}\right) .
$$

If this limit exists, then the element $d s$ is said to induce a cone on $\left.S^{1} \times\right] a, b[$ with apex at $b$. The nonnegative number $\alpha$ is called the angle of the cone.

A hyperbolic cusp is a cone with angle zero. Indeed, the (model) hyperbolic cusp is the half-infinite cylinder, $\left.S^{1} \times\right] 0, \infty$ [ equipped with the metric $m_{0}=$ $y^{-2}\left(d \theta^{2}+d y^{2}\right)$. One computes that $\ell(y)=y^{-1}$ and $d\left(y_{1}, y_{2}\right)=\left|\log \left(y_{1} / y_{2}\right)\right|$, and hence the limit - as $y$ tends to $\infty$ - in (1) exists and is equal to zero. Note that the apex of this cone is at $y=\infty$.

The metric $m_{0}$ has constant curvature -1 , hence the term hyperbolic. In general, a metric of the form $m=\rho^{-2}\left(d \theta^{2}+d y^{2}\right)$ on $\left.S^{1} \times\right] 0, \infty$, is hyperbolic iff $-\Delta \log \rho=$ $\rho^{-2}$ where $\Delta=\partial_{\theta}^{2}+\partial_{y}^{2}$ is the Euclidean Laplacian [Ahl]. If $\rho$ is $\theta$-invariant, then the hyperbolicity of $m$ is equivalent to the satisfaction of $\left(\rho_{y}\right)^{2}-\rho_{y y} \cdot \rho=1$. A direct computation shows that this equation is satisfied by $\rho_{\alpha}(y)=\alpha^{-1} \sinh (\alpha y)$ where $\alpha \neq 0$.

Define $m_{\alpha} \equiv \rho_{\alpha}^{-2}\left(d \theta^{2}+d y^{2}\right)$. One checks that $\left(S^{1} \times\right] 0, \infty\left[, m_{\alpha}\right)$ is a cone of angle $\alpha$ with apex at $y=\infty$. By design $m_{\alpha}$ is hyperbolic, and hence we call $\left(S^{1} \times\right] 0, \infty\left[, m_{\alpha}\right)$ the model hyperbolic cone of angle $\alpha{ }^{1}$ The function $\alpha \rightarrow \rho_{\alpha}$ extends uniquely to an entire function. In particular, $m_{\alpha}$ is a real-analytic family of metrics that conformally converts a cusp into a cone.

\section{THE IMPLICIT FUNCTION THEOREM ARGUMENT}

For simplicity of exposition we assume that $M$ is a surface with one (cylindrical) $\mathrm{end}^{2} E \subset M$. The generalization to a finite number of ends is straightforward. We consider hyperbolic metrics $g$ on $M$ such that $(E, g)$ is a cone. In [Jdg93] it is shown that there is an isometric embedding of $(E, g)$ into a model hyperbolic cone with the same angle.

The Gauss-Bonnet formula and the negativity of the curvature together imply that the cone angle $\alpha$ of $(E, g)$ satisfies $\alpha-2 \pi+2 \pi \cdot \chi(M)<0$ where $\chi(M)$ is the Euler characteristic. R. McOwen [McO88] has shown that this angular condition is also sufficient for the existence of such a metric within an arbitrary conformal equivalence class of metrics. Moreover, McOwen has proven that the metric is determined by its (pointwise) conformal structure together with the angle $\alpha$. Thus, we have a bijection $\alpha \rightarrow g_{\alpha}$ from the interval $I(M)=[0,2 \pi(1-\chi(M))$ [ onto the set of conical hyperbolic metrics in a given conformal equivalence class.

\footnotetext{
${ }^{1}$ An equivalent description of a model hyperbolic cone: The isometry group of the universal cover, $U$, of the once-puncture hyperbolic plane is isomorphic to $(\mathbb{R},+)$. This isomorphism is normalized by requiring that the hyperbolic plane is recovered as the quotient $U /<2 \pi\rangle$. The model hyperbolic cone of angle $\alpha>0$ is the quotient $U /\langle\alpha\rangle$.

${ }^{2}$ Here we use the term 'end' to abbreviate the phrase 'neighborhood of an end'.
} 
In what follows, we fix a (pointwise) conformal structure and an initial angle $\alpha_{0} \in I(M)$. For each $\alpha \in I(M)$, there is a unique function $w_{\alpha}: M \rightarrow \mathbb{R}$ defined by $g_{\alpha}=e^{2 w_{\alpha}} \cdot g_{\alpha_{0}}$. The function $w_{\alpha}$ is known to satisfy [Ahl] [KzdWrn74]

$$
\Delta_{\alpha_{0}} w_{\alpha}-e^{2 w_{\alpha}}+1=0
$$

where $\Delta_{\alpha_{0}}=+\operatorname{div}(\mathrm{grad})$ is the Laplacian associated to $g_{\alpha_{0}} \cdot{ }^{3}$ Conversely, a solution to (2), satisfying certain asymptotics in the cylindrical end $E$, gives rise to a hyperbolic metric $g$ such that $(E, g)$ is a cone [McO88]. In other words, $\left\{w_{\alpha}: \alpha \in I(M)\right\}$ is the zero level set of the operator $f \rightarrow \Delta_{\alpha_{0}} f-e^{2 f}+1$. The $f$-derivative, $\Delta_{\alpha_{0}}-2$, is 'invertible' in the sense that $\left.\left.2 \notin \operatorname{spec}\left(\Delta_{\alpha_{0}}\right) \subset\right]-\infty, 0\right]$. The (analytic) implicit function theorem can now be invoked.

Of course, the preceding argument is only heuristic. To make this argument rigorous, it remains to redefine the operator with explicit domain and range, make the dependence on $\alpha$ explicit, and prove the invertibility of the derivative. All of these details are interlinked. The invertibility of the derivative is a particularly important constraint on the choice of definitions that we make below.

The dependence on $\alpha$ is contained in the relative asymptotics of the solutions to (2). We define a function $v_{\alpha}: M \rightarrow \mathbb{R}$ that describes these asymptotics. First, let $\left.\phi: E \rightarrow S^{1} \times\right] 0, \infty\left[\right.$ be the embedding such that $\phi^{*}\left(m_{\alpha_{0}}\right)=g_{\alpha_{0}}$. Let $\chi$ be a smooth function on $M$ that is equal to 1 on a nonempty proper subcone of $\left(E, g_{\alpha_{0}}\right)$ and equal to zero on $M \backslash E$. Define $\bar{\rho}_{\alpha} \in C^{\infty}(M)$ by

$$
\bar{\rho}_{\alpha}(m)=\left\{\begin{array}{cl}
\exp \left(\chi(m) \cdot \log \left(\rho_{\alpha} \circ \phi^{-1}\right)(m)\right), & m \in E, \\
1, & m \in M \backslash E,
\end{array}\right.
$$

where $\rho_{\alpha}$ is defined as in the previous section. Define $v_{\alpha}=\log \left(\bar{\rho}_{\alpha_{0}} / \bar{\rho}_{\alpha}\right)$.

We incorporate the dependence on $\alpha$ by defining an operator whose zero level set contains $w_{\alpha}-v_{\alpha}$. First consider the map $F$ defined by $F(\alpha, u)=\Delta_{\alpha_{0}}\left(u+v_{\alpha}\right)-$ $e^{2\left(u+v_{\alpha}\right)}+1$. For $u \in C^{2}(M)$ with support belonging to $E$, we have:

$$
\begin{aligned}
F(\alpha, u) & =\Delta_{\alpha_{0}} u+\Delta_{\alpha_{0}} \log \left(\frac{\rho_{\alpha_{0}}}{\rho_{\alpha}}\right)-e^{2 u}\left(\frac{\rho_{\alpha_{0}}}{\rho_{\alpha}}\right)^{2}+1 \\
& =\Delta_{\alpha_{0}} u+\left(1-e^{2 u}\right)\left(\frac{\rho_{\alpha_{0}}}{\rho_{\alpha}}\right)^{2} .
\end{aligned}
$$

Here we have suppressed the embedding $\phi: E \rightarrow] 0, \infty[$ from notation. The function $\bar{\rho}_{\alpha_{0}} / \bar{\rho}_{\alpha}$ is unbounded for $\alpha<\alpha_{0}$, and hence we replace $F$ with $G$ defined by $G(\alpha, u)=\left(\bar{\rho}_{\alpha_{0}}\right)^{-2} \cdot F(\alpha, u)$. For $u \in C^{2}(M)$ with support in $E$ we have

$$
G(\alpha, u)=\Delta_{E u c} u+\left(1-e^{2 u}\right)\left(\rho_{\alpha}\right)^{-2},
$$

where $\Delta_{E u c}=\partial_{\theta}^{2}+\partial_{y}^{2}$ is the Euclidean Laplacian.

\section{Some HÖLDER SPACES}

For the domain and range of $G(\alpha, \cdot)$, we use a variant of the usual Hölder spaces. ${ }^{4}$ Let $(M, g)$ be a Riemannian manifold and let dist denote the associated distance

\footnotetext{
${ }^{3}$ In the present paper, the sign convention for the Laplacian makes it a nonpositive operator.

${ }^{4}$ One could also try using Sobolev spaces. However, the (relative) integrability of $u$ appears to give very little control on $e^{u}$ far out in the cylinder. (Local estimates on $u \rightarrow e^{2 u}$ are given by the Trudinger inequality, [KzdWrn74].)
} 
function. Let $r: \mathbb{R} \rightarrow \mathbb{R}$ be defined by

$$
r(x)= \begin{cases}x, & x \leq 1 \\ 1, & x \geq 1\end{cases}
$$

Define for $f: M \rightarrow \mathbf{C}$ and $0 \leq \beta<1$

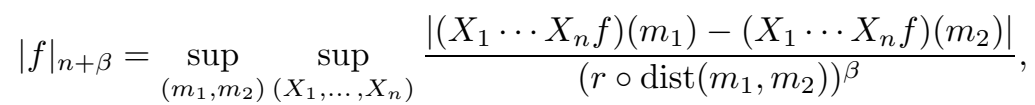

where $\left(m_{1}, m_{2}\right) \in M \times M$ and $\left(X_{1}, \ldots, X_{k}\right)$ runs over all $k$-tuples of vector fields $X_{i}$ with $g\left(X_{i}, X_{i}\right)=1, k=0,1, \ldots, n$. For $0<\beta<1$, define $C^{n+\beta}(M, g)$ to be the Banach space of functions that are finite with respect to the norm $\|f\|_{C^{n+\beta}(M, g)} \equiv$ $\max \left\{|f|_{n+0},|f|_{n+\beta}\right\}$.

Lemma 1. Let $(M, g)$ be a Riemannian manifold. If $\beta^{\prime}>\beta$, then $C^{k+\beta^{\prime}}(N)$ is compact in $C^{k+\beta}(N)$. Suppose that $(M, g)$ has finite diameter, $\operatorname{diam}(M, g)$, positive injectivity radius, $\operatorname{inj}(M, g)$, upper and lower bounds on its sectional curvature, and smooth boundary. Let $L$ be a uniformly elliptic ${ }^{5}$ second order differential operator on $(M, g)$. Then a Schauder type estimate holds: There exists $C>0$ such that for any $f \in C^{k+2}(M, g)$

$$
\|f\|_{(k+2)+\alpha} \leq C\left(\|f\|_{k+0}+\|f\|_{(k+2)+\alpha}^{\partial M}+\|L f\|_{k+\alpha}\right) .
$$

Here $\|\cdot\|_{n+\alpha}^{\partial M}$ denotes the norm associated to the boundary of $M$ with the induced metric. The constant $C$ depends only on the ellipticity constant of $L$, an upper bound on $\operatorname{diam}(M, g)$, a lower bound on $\operatorname{inj}(M, g)$, upper and lower bounds on the curvature of $(M, g)$, and $C^{\alpha}$ bound on the coefficients of $L$.

Proof. Note that by exhausting $M$ with an appropriate sequence of open submanifolds and applying a diagonalization argument, one reduces the first part of the Lemma to the case represented by the hypotheses of the second part. Namely, it suffices to assume that $(M, g)$ has finite diameter, positive injectivity radius, and upper and lower bounds on its sectional curvature.

Let $\mathcal{B}$ be the collections of balls in $(M, g)$ with radius $\rho=\operatorname{inj}(M, g)$. We claim that for all $f \in C^{k+\alpha}(M, g)$ we have

$$
\frac{\operatorname{inj}(M, g)}{\operatorname{diam}(M, g)}\|f\|_{k+\alpha} \leq \sup _{B \in \mathcal{B}}\left\|\left.f\right|_{B}\right\|_{k+\alpha}^{B} \leq \sup \|f\|_{\alpha}
$$

where $\|\cdot\|_{k+\alpha}^{B}$ denotes the norm associated to $C^{k+\alpha}(B, g)$. The 'nonobvious' part of (9) is the (pointwise version of the) inequality on the left hand side in the case where $m_{1}$ and $m_{2}$ of $(7) \operatorname{satisfy} \operatorname{dist}\left(m_{1}, m_{2}\right)>2 \cdot \operatorname{inj}(M, g)=2 \rho$. In this case, one lets $0<\epsilon<\operatorname{inj}(M, g)$ and considers a curve $\gamma$ joining $m_{1}$ and $m_{2}$ having length equal to $\operatorname{dist}\left(m_{1}, m_{2}\right)+\epsilon$. An elementary argument shows that there is a finite set of points $\left\{x_{1}, \ldots, x_{n}\right\} \subset \gamma$ with $x_{1}=m_{1}, x_{n}=m_{2}, 2 \rho>\operatorname{dist}\left(x_{i}, x_{i+1}\right)>\rho$, and $n<\rho^{-1} \cdot \operatorname{dist}\left(m_{1}, m_{2}\right)+\epsilon$. One then has (in the case $k=0$ )

$$
\begin{aligned}
\frac{\left|f\left(m_{1}\right)-f\left(m_{2}\right)\right|}{\left(r \circ \operatorname{dist}\left(m_{1}, m_{2}\right)\right)^{\alpha}} & \leq \sum_{i=1}^{n} \frac{\left|f\left(x_{i-1}\right)-f\left(x_{i}\right)\right|}{\left(r \circ \operatorname{dist}\left(x_{i-1}, x_{i}\right)\right)^{\alpha}} \\
& \leq\left.\left(\frac{\operatorname{dist}\left(m_{1}, m_{2}\right)+\epsilon}{\rho}\right) \sup _{B \in \mathcal{B}}|| f\right|_{B} \|_{\alpha}^{B} .
\end{aligned}
$$

The claim follows from letting $\epsilon$ tend to zero.

\footnotetext{
${ }^{5}$ The ellipticity constant should be defined in terms of unit vector fields on $(M, g)$.
} 
The exponential map associated to the point $m \in M$ may be regarded as a diffeomorphism $\phi_{m}$ from the Euclidean ball $\left(D, g_{0}\right)$ of radius $\rho$ onto the ball $B \in \mathcal{B}$ centered at $m$. Since the sectional curvatures are uniformly bounded, there is a constant $C>1$ such that for all $m \in M$, the pull-back map $\phi_{m}^{*}: C^{k+\alpha}(B, g) \rightarrow$ $C^{k+\alpha}\left(D, g_{0}\right)$ satisfies $C^{-1}\|f\|_{k+\alpha} \leq\left\|\phi^{*}(f)\right\|_{k+\alpha} \leq C\|f\|_{k+\alpha}$. By using this and (9), one obtains the Lemma from the standard compactness theorem and Schauder estimate [BJS].

The following refinement of (9) will also prove useful.

Lemma 2. Let $(M, g)$ be a Riemannian manifold. Let $\mathcal{D}$ be an open cover of $M$ such that for any two points $m_{1}, m_{2} \in M$ with $\operatorname{dist}\left(m_{1}, m_{2}\right) \leq 1$ there is a $D \in \mathcal{D}$ such that $m_{1}, m_{2} \in D$. Then for every $f \in C^{k+\alpha}(M, g)$ we have

$$
\|f\|_{k+\alpha}=\max \left\{\|f\|_{k+0}, \sup _{D \in \mathcal{D}}\left\|\left.f\right|_{D}\right\|_{k+\alpha}^{D}\right\}
$$

where $\|\cdot\|_{k+\alpha}^{D}$ denotes the norm associated to $C^{k+\alpha}(D, g)$.

Proof. It follows from the definitions that the left hand side of (12) is greater than or equal to the right hand side. As for the remaining inequality: Note that if $\operatorname{dist}\left(m_{1}, m_{2}\right) \geq 1$, then $r \circ \operatorname{dist}\left(m_{1}, m_{2}\right)=1$, and otherwise we may find a $D \in \mathcal{D}$ with $m_{1}, m_{2} \in D$. The inequality then follows from inspection of (7).

\section{REAL-ANALYTiCITY OF THE METRIC FAMiLY}

We are now ready to prove:

Theorem 1. Let $M$ be a surface having one end $E$. Let $[g]$ be a pointwise conformal class of hyperbolic metrics on $M$. For each $\alpha \in\left[0,2 \pi(1-\chi(M))\left[\right.\right.$, let $g_{\alpha}$ be the unique hyperbolic metric tensor on $M$ such that $\left(E, g_{\alpha}\right)$ is a cone of angle $\alpha$. Define the conformal factor $w_{\alpha}$ by $g_{\alpha}=e^{w_{\alpha}} \cdot g_{0}$. Then the map $\alpha \rightarrow w_{\alpha} \in C^{\infty}(M)$ is real-analytic.

Proof. Let $h$ be the Riemannian metric $\left(\bar{\rho}_{\alpha_{0}}\right)^{2} \cdot g_{\alpha_{0}}$. Recall that for functions with support near infinity in $E$, the operator $G$ takes the form given in (5). Near infinity, $(E, h)$ is isometric to a half-infinite Euclidean cylinder. It follows that $G$ sends $\mathbf{C} \times Y$ into $Z$ where $Y=C^{n+2+\alpha}(M, h)$ and $Z=C^{n+\alpha}(M, h) .{ }^{6}$ By the discussion in the previous section, it suffices to verify the hypotheses of a Banach space analytic implicit function theorem [Brg] for the map $G$.

The function $\bar{\rho}_{\alpha}$ depends analytically on $\alpha \in \mathbf{C}$, the exponential map $u \rightarrow e^{2 u}$ has a convergent power series, and $\Delta_{h}$ is linear. It follows that $G$ is a complex analytic map of Banach spaces. It remains to verify the following lemma.

Lemma 3. The Frechet derivative, $d G_{Y}\left(\vec{\alpha}_{0}, 0\right): Y \rightarrow Z$, has a bounded inverse.

Proof. A computation gives

$$
d G_{Y}\left(\alpha_{0}, 0\right)=\left(\bar{\rho}_{\alpha_{0}}\right)^{-2}\left(\Delta_{\alpha_{0}}-2\right) .
$$

Our definition of Hölder space forces every Hölder continuous function to be bounded on $M$. Thus, since $\left(M, g_{\alpha_{0}}\right)$ has finite volume, we have the inclusion $Y \subset L^{2}\left(M, g_{\alpha_{0}}\right)$. The operator $\Delta_{\alpha_{0}}$ is nonnegative on $L^{2}\left(M, g_{\alpha_{0}}\right)$ and hence 2 cannot be an eigenvalue. In sum, the null space of $d G_{Y}: Y \rightarrow Z$ is trivial, and thus $d G_{Y}$ is injective.

\footnotetext{
${ }^{6}$ Here $\alpha$ is complex. In the end, we restrict $\alpha$ to $[0,2 \pi(1-\chi(M))[$.
} 
Given $f \in Z$ we wish to find $\psi \in Y$ so that

$$
\left(\bar{\rho}_{\alpha_{0}}\right)^{-2}\left(\Delta_{\vec{\alpha}_{0}}-2\right) \psi=f .
$$

Let $\phi: E \rightarrow] 0, \infty\left[\right.$ be the embedding defined in $\S 3$. Let $M_{a}$ denote the set $M \backslash \phi^{-1}\left(S^{1} \times\left[a, \infty[)\right.\right.$. The manifold $\left(M_{a}, h\right)$ has finite diameter, bounded curvature, and positive injectivity radius. The operator $d G_{Y}\left(\alpha_{0}, 0\right): C^{2+\alpha}\left(M_{a}, h\right) \rightarrow$ $C^{0+\alpha}\left(M_{a}, h\right)$ is uniformly elliptic with $C^{\alpha}$-bounded coefficients. Thus, Lemma 1 applies, and we may use the continuity method [BJS] to find a solution $\psi_{a}$ on $M_{a}$ to (13) that vanishes on the boundary $\partial M_{a}$.

For each $a \in] 0, \infty\left[\right.$, consider the sequence $\left\{\psi_{b}\right\}_{b \geq a}$. By the maximum principle,

$$
\left|\psi_{b}\right|_{C^{0}\left(M_{a}\right)} \leq \frac{1}{2}|f|_{C^{0}(M)}
$$

for all $b>a$. Applying (8) to $\left(M_{a}, h\right)$ with $L=d G_{Y}\left(\alpha_{0}, 0\right)$ we have $C_{a}$ such that if $b \geq a$, then $\left\|\psi_{b}\right\|_{C^{n+2+\beta}\left(M_{a}\right)} \leq C_{a}$. Then Lemma 1 implies that for $\beta^{\prime}<\beta$, the sequence $\left\{\psi_{b}\right\}_{b \geq a}$ has a $C^{n+2+\beta^{\prime}}$-convergent subsequence. A diagonalization argument $(a \rightarrow \infty)$ provides a limit $\psi$ that belongs to $C^{n+2+\beta^{\prime}}\left(M_{a}\right)$ for each $a \in$ ] $0, \infty[$.

By continuity, $\psi$ satisfies (13), and therefore $\psi \in Z$ by regularity. Further, the bound (14) applies to $\psi$, and hence

$$
|\psi|_{C^{0}(M)} \leq \frac{1}{2}|| f \|_{Z}
$$

To finish the proof, we must show that $\|\psi\|_{Y} \leq C\|f\|_{Z}$ where $C$ is a constant that does not depend on $f$. For each $a$, let $S_{a}$ denote the interior of the set $M_{a+2} \backslash M_{a}$. Fix $a_{0}$ and suppose that $\operatorname{dist}\left(m_{1}, m_{2}\right) \leq 1$. Then either both $m_{1}$, $m_{2} \in M_{a_{0}}$ or there is some $a$ such that both $m_{1}, m_{2} \in S_{a}$. Thus, by Lemma 2 it will suffice to show that there is a constant $C^{*}$, independent of $a$, such that $\|\psi\|_{n+2+\beta}^{S_{a}} \leq\|f\|_{n+\beta}^{S_{a}}$.

It is crucial to note that for $a$ large enough, $\left(S_{a}, h\right)$ is isometric to the Euclidean cylinder $\left(S^{1} \times[0,1], d \theta^{2}+d y^{2}\right)$. In particular, the injectivity radius, the diameter, the curvature, and the smoothness of the boundary of $\left(S_{a}, h\right)$, are all independent of $a$. The operator $d G_{Y}$ restricted to $C^{n+2+\beta}\left(S_{a}, h\right)$ is equal to $\Delta_{E u c}-2\left(\bar{\rho}_{\alpha_{0}}\right)^{-2}$. For a given $a$, we have from (8):

$$
\|\psi\|_{n+2+\beta}^{S_{a}} \leq C^{*}\left(\|f\|_{n+\beta}^{S_{a}}+\|\psi\|_{n+0}^{S_{a}}\right) .
$$

The constant $C^{*}$ depends only on the geometry of the domain (which is constant), the ellipticity constant of the operator $\Delta_{E u c}-2\left(\bar{\rho}_{\alpha_{0}}\right)^{-2}$ (which is constant), and the $C^{\alpha}$ bounds on the coefficients of the operator. Since the smooth function $\left(\bar{\rho}_{\alpha_{0}}\right)^{-2}$ decays at $\infty$, the constant $C^{*}$ can be chosen to be independent of $\left.a \in\right] 0, \infty[$.

\section{The Angular SECOND DERIVATive}

Let $E_{s}: M \rightarrow \mathbf{C}$ denote the Eisenstein series associated to the cusped hyperbolic surface $\left(M, g_{0}\right)$ having eigenvalue $s(s-1)[\mathrm{Vnk}] .^{7}$

Theorem 2. Let $K \subset M$ be compact. The conformal factor $w_{\alpha}$ satisfies

$$
d\left(w_{\alpha}, \frac{\alpha^{2}}{6} \cdot E_{2}\right)=O\left(\alpha^{4}\right)
$$

\footnotetext{
${ }^{7}$ Recall that our choice of sign makes the Laplacian a nonpositive operator.
} 
where $d$ is the metric for the Frechét space $C^{\infty}(M)$.

Proof. The above constructions are invariant under $\alpha \rightarrow-\alpha$. In particular, $\alpha \rightarrow \rho_{\alpha}$ is an even function. Thus $G(\alpha, \cdot), v_{\alpha}$, and hence $w_{\alpha}$, are all even functions of $\alpha \in \mathbb{R}$. Moreover, $w_{0}=v_{0} \equiv 0$. Hence, the respective power series about $\alpha=0$ take the

form $\sum_{k>0} \alpha^{2 k} v_{2 k}$ and $\sum_{k>0} \alpha^{2 k} w_{2 k}$. By substituting the expansion for $w_{\alpha}$ into (2) and combining like terms in $\alpha$, we find that $\left(\Delta_{\alpha_{0}}-2\right) w_{2}=0$.

In the proof of Theorem 1, we proved that the path $\alpha \rightarrow\left(w_{\alpha}-v_{\alpha}\right)$ is analytic in $C^{n+\beta}(M, h)$. In particular, $w_{2}-v_{2}$ is bounded, and thus $w_{2}$ is asymptotic to $v_{2}$ on the cylinder $E$. By (3), the function $v_{\alpha}$ restricted to a subcone of $E$ is equal to

$$
v_{\alpha}=-\log \left(\frac{\sinh (\alpha y)}{\alpha y}\right)=\left(\frac{\alpha^{2}}{6}\right) \cdot y+\sum_{k>1} \alpha^{2 k} v_{2 k} .
$$

In sum, $w_{2}$ is asymptotic to $\alpha^{2} y / 6$ as $y$ tends to infinity. The function $E_{2}$ is the unique function that is asymptotic to $y^{2}$ satisfying $\Delta_{0} E_{2}=2 \cdot E_{2}$ [CdV83].

Remark 1. The Eisenstein series $E_{2}$ has a fairly explicit description. Let $\Gamma \subset$ $S L(2, \mathbb{R})$ be a uniformization group of $\left(M, g_{0}\right)$ such that the cusp corresponds to (orbit of) the ideal point at $\infty$. Let $\Gamma_{\infty}$ be the stabilizer of $\infty$. Then

$$
E_{2}(z)=\sum_{[\gamma] \in \Gamma / \Gamma_{\infty}}(\operatorname{Im}(\gamma \cdot z))^{2}
$$

Remark 2. In the preceding theorem, we considered the conformal difference, $w_{\alpha}$, between $g_{\alpha}$ and $g_{0}$, and then expanded about $\alpha=0$. One can also consider the conformal difference between $g_{\alpha}$ and $g_{\alpha_{0}}$ and then expand about $\alpha_{0}$. In the latter case, the first order term in the power series expansion is $\alpha \cdot \lim _{m^{\prime} \rightarrow \infty} K\left(m^{\prime}, m\right)$ where $K$ is the Schwartz kernel of $(\Delta-2)^{-1}$.

\section{REFERENCES}

[Ahl] Ahlfors, L., Conformal Invariants: Topics in geometric function theory, McGrawHill (1973). MR 50:10211

[Brg] Berger, M.S., Nonlinearity and Functional Analysis, Academic Press, New York (1977).

[BJS] Bers, L., John, F., and Shechter, M., Partial Differential Equations, Amer. Math. Soc. (1964). MR 82c:35001

[CdV83] Colin de Verdiere, Y., Pseudo-laplaciens II, Ann. Inst. Fourier 33 (1983), 87-113. MR 84k:58222

[Jdg93] Judge, C., Thesis, Univ. of Maryland (1993).

[Jdg95] Judge, C. On the Existence of Maass cusp forms on hyperbolic surfaces with cone points, J. Amer. Math. Soc. 8 (1995), 715-759. MR 96b:11069

[KzdWrn74] Kazdan, J. and Warner, F., Curvature functions for compact 2-manifolds, Ann. of Math. 99 (1974), 14-47. MR 49:7949

[McO88] McOwen, R., Point singularities and conformal metrics on Riemann surfaces, Proc. Amer. Math. Soc. 103 (1988), 222-224. MR 89m:30089

[Vnk] Venkov, A.B., The Spectral Theory of Automorphic Functions, Klüwer (1990). MR 93a: 11046

[Wlf91] Wolf, M., Infinite energy harmonic maps and degeneration of hyperbolic surfaces in moduli space, J. Diff. Geom. 33 (1991), 487-539. MR 92b:58055

Indiana University, Bloomington, Indiana

E-mail address: cjudge@poincare.math.indiana.edu

$U R L:$ http://poincare.math.indiana.edu/ ${ }^{c j u d g e}$ 